\title{
Guest Editorial: Online Experimentation for Industrial, Health and Educational Purposes
}

\author{
https://doi.org/10.3991/ijoe.v16i04.13883 \\ Alberto Cardoso \\ CISUC, Dep. of Informatics Engineering, University of Coimbra, Portugal \\ alberto@dei.uc.pt \\ Maria Teresa Restivo \\ LAETA - INEGI, Faculty of Engineering, University of Porto, Porto, Portugal \\ trestivo@fe.up.pt
}

The Special Issue of the International Journal of Online and Biomedical Engineering (iJOE) is publishing extended versions of interesting papers presented at the Experiment@International Conference 2019 (exp.at'19), dedicated to online experimentation.

Within iJOE, Vol.9 (2013): Special Issue expat'13, the concept of Online Experimentation was proposed. It encloses "remote access to experiments, virtual $2 \mathrm{D}$ and $3 \mathrm{D}$ simulated experiments, augmented and virtual reality apps, all combined with sensorial devices for remote or virtual reality interaction, potentiated by additional tools, available through web collaborative platforms for exploring and increasing the user immersion in real systems or in their virtual replicas."

This basilar concept expanded expat' $x \boldsymbol{x}$ conference series in a forum for "engineers, researchers and industry and health professionals, to promote interactions and to contribute to science and technology developments in remote and virtual experiments, smart devices, communication protocols, web interface design, collaborative digital environments and virtual and augmented reality, haptics and sensorial device interactions", in line with Industry 4.0 perspectives.

All the engineering fields, industries, health area, entertainment, business, military and security, integrate online experimentation supporting technologies. Therefore, a diversified set of special sessions were submitted to expat'19, giving rise to distinct contributions for this special issue. "Automotive control and online experimentation in control, air quality monitoring, remote and digital pneumatics, ocean monitoring, immersive systems and sensorial stimulation, machine learning applications in engineering, smart technologies and interactive media for health, online resources in biomedical engineering and online experimentation in science and engineering education" were topics promoted by special sessions at exp.at'19.

The articles included in this Special Issue comprise different perspectives of online experimentation, showing the diversity and actuality of this area.

The use of new information and IoT technologies in the context of the Industry 4.0 paradigm opens up several challenges and opportunities to improve industrial production processes, aircraft maintenance operations and air quality monitoring and forecasting tasks. 
Three articles present works developed in these topics:

- Development of a Smart Gateway for a Label Loom Machine using Industrial IoT Technologies

- Web-based tool for the simulation of a Prognostics and Health Management model Prediction of the Remaining Useful Life of Aircraft Components

- NanoSen-AQM: From Sensors to Users.

The development of resources and applications of online experimentation in the health field has been increasing significantly in recent years, showing that augmented and virtual reality, as well as augmented spaces, eventually combined with serious games, can be successfully considered for different valences, such as psychotherapy, therapeutic rehabilitation or mental health.

Three contributions illustrate works in these areas:

- An Adaptive Virtual Reality-Based Serious Game for Therapeutic Rehabilitation

- Towards Accessible Mental Healthcare through Augmented Reality and SelfAssessment Tools

- Augmented Shared Spaces: An Application for Exposure Psychotherapy.

The availability of increasingly powerful tools based on machine learning and serious games, offers effective conditions to support human-computer interactions, namely in terms of recognizing human emotion through speech and training of spelling skills.

Two articles present works in this area:

- Speaker Awareness for Speech Emotion Recognition

- Child friendly app for spelling training.

Several international projects have been carried out for the development of different architectures, frameworks and platforms based on remote and virtual laboratories, demonstrating the usefulness of online experimentation, for example, for educational purposes.

An article describes the contribution of a project for the definition of a grid of laboratories, which can be shared and accessed by several users in different contexts of use:

- A Federation of Remote Laboratory OERs Grid of VISIR systems through PILAR Project.

This set of articles illustrates the potential of resources based on online experimentation for diverse application areas, such as industrial, health and educational purposes.

\section{Authors}

Alberto Cardoso is with CISUC, Department of Informatics Engineering of the University of Coimbra, Coimbra, Portugal (email: alberto@dei.uc.pt).

Maria Teresa Restivo is with LAETA - INEGI, Faculty of Engineering, University of Porto, Porto, Portugal. (email: trestivo@fe.up.pt). 